\title{
Modified Elman Neural-PID Controller Design for DC-DC Buck Converter System Based on Dolphin Echolocation Optimization
}

\author{
Khulood E. Dagher \\ Al-Khwarizmi Collage of Engineering /University of Baghdad/Baghdad/Iraq \\ Email: dagherkhulood@kecbu.uobagdad.edu.iq
}

(Received 25 September 2017; accepted 21 January 2018)

https://doi.org/10.22153/kej.2018.01.009

\begin{abstract}
This paper describes a new proposed structure of the Proportional Integral Derivative (PID) controller based on modified Elman neural network for the DC-DC buck converter system which is used in battery operation of the portable devices. The Dolphin Echolocation Optimization (DEO) algorithm is considered as a perfect on-line tuning technique therefore, it was used for tuning and obtaining the parameters of the modified Elman neural-PID controller to avoid the local minimum problem during learning the proposed controller. Simulation results show that the best weight parameters of the proposed controller, which are taken from the DEO, lead to find the best action and unsaturated state that will stabilize the Buck converter system performance and achieve the desired output. In addition, there is a minimization for the tracking voltage error to zero value of the Buck converter output, especially when changing a load resistance by $10 \%$.
\end{abstract}

Keywords: Buck Converter, Dolphin Echolocation, Modified Elman Neural PID Controller, On-Line-Tuning Optimization.

\section{Introduction}

DC-DC buck convertor is a very versatile electronic circuit due to its essential use in battery operations for different applications such as computer systems, portable devices, office equipment and any other devices that need to get an output voltage more or less than the input voltage. Buck convertors acquired their name from the reality of their work, where the amplitude of input voltage is bucked/chopped or decreased to get the output voltage [1]. In fact, the optimal or near optimal control of the attenuation operation is not easy and this is because of the load variation thus, we can find various researches that solve this problem and the backbone for these researches is the famous PID controller which is well known by its structure simplicity, time domain regulation and good closed loop response [2]. Hence, many optimization methods are developed to find the control gain parameters for the PID controller that is used to control the buck convertor, and some of these methods are: [3] presented a fuzzy PID controller with no defuzzification module. In [4] the authors proposed a new adaptive method based on simulated annealing algorithm to control the output voltage of the convertor.

In [5] the authors proposed an on-line tuning method based on particle swarm optimization and they show high control performance. In addition to that, the genetic algorithm as a tuning control algorithm for buck converter is used as in [6]. This paper presents a new structure of the controller that uses a modified Elman neural network in the form of the PID controller equation and the control parameters are tuned by an optimization algorithm named as Dolphin Echolocation Optimization DEO algorithm in order to get fast, accurate and robust control action for the DC buck converter. 
DEO was presented by Ali in 2013 [7] and proved as the most powerful optimization meta-huristic method as compared with other evolutionary algorithms in terms of the number of function evaluations and in convergence toward reaching the optimal solution.

The description of this paper is as follows: Section two describes the mathematical model of the DC-DC Buck converter. Section three explains the design of the proposed modified Elman Neural-PID controller. In section four, the Dolphin echolocation optimization algorithm is explained. Section five presents the simulation results of the proposed controller. Finally, the conclusions are explained in section six.

\section{Buck Converter Circuit Model}

In this work, an asynchronous Buck convertor model is used with two n-channel MOSFETs as shown in Figure (1) to make the output voltage $\mathrm{V}_{\text {out }}$ always less than the supply voltage Vs.

This converter uses two controllable switches to achieve its operation rather than using one nchannel MOSFET power switch and one power diode rectifier, thus it has a maximize conversion efficiency and fast switching transient $[4,8]$.

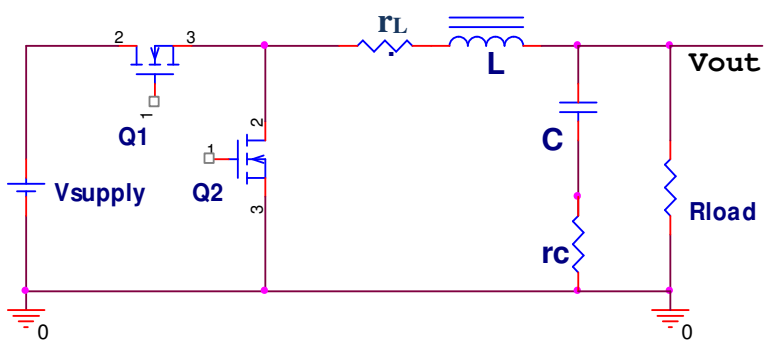

Fig. 1. Schematic diagram of Synchronous Buck power stage [8].

In general, the operation of the Buck converter depends on $\mathrm{Q}_{1}$ of the MOS switch and $\mathrm{Q}_{2}$ of the MOS synchronous rectifier. The parameter values of the buck convertor taken from $[3,4]$ is shown in Table 1.

Now to drive and analyze the mathematical model of the buck convertor two operation time have to be considered depending on two MOSFETs (Q1 and Q2) [3, 4] as follows:

- At period of time $\mathrm{t}<\mathrm{T} 1$, Switching ON Q1 and switching OFF Q2:

The circuit analysis can be illustrated by applying Kirshoff's voltage law as follows:

$$
V_{S}-i_{L} \cdot r s_{\text {on }}-L \frac{d i_{L}}{d t}-i_{L} \cdot r_{L}-V_{\text {oUT }}=0
$$

Where $V_{S}$ : is the supply voltage; $i_{L}$ : is the inductor current; $r s_{o n}$ : is the on $\mathrm{n}$-channel resistance of the MOSFET; $r_{L}$ : is inductor effective series resistance; $V_{\text {out }}$ : is the output voltage of the circuit.

At period of time $\mathrm{T} 1<\mathrm{t}<\mathrm{T} 2$, Switching OFF Q1 and switching ON Q2:

The circuit analysis can be illustrated by applying Kirshoff's voltage law as follows:

$-i_{L} \cdot r s_{\text {on }}-L \frac{d i_{L}}{d t}-i_{L} \cdot r_{L}-V_{\text {oUT }}=0$

By defining $\Delta$ as Eq. (3), as a switching function to turn $\mathrm{V}_{\text {Supply }} \mathrm{ON}$ and OFF:

$$
\Delta=\left\{\begin{array}{lc}
1 & 0<t<T_{1} \\
0 & T_{1}<t<T_{2}
\end{array}\right.
$$

So the mathematical model (Eq. (1) and Eq. (2)) can be written in state space representation as follows:

Let:

$x_{l}=i_{L}, \quad \dot{x}_{1}=\frac{d i_{L}}{d t}, x_{2}=V_{\text {OUT }}, \dot{x}_{2}=\frac{d V_{\text {OUT }}}{d t}$

$\left.\begin{array}{l}\dot{x} \\ y\end{array}=\begin{array}{c}A x+B V_{S} \\ C x\end{array}\right\}$

$A=\left[\begin{array}{ll}a_{11} & a_{12} \\ a_{21} & a_{22}\end{array}\right]=$

$\left[\begin{array}{cc}\frac{-\left(r s_{o n}+r_{C}\right)}{L} & \frac{-1}{L} \\ \frac{-\left(r s_{O n}+r_{L}\right)\left(C R_{L}+r_{C}\right)}{L C\left(R_{L}+r_{C}\right)} & \frac{-\left(C R_{L}+r_{C}\right)}{L C\left(R_{L}+r_{C}\right)}\end{array}\right]$

$B=\left[\begin{array}{l}b_{1} \\ b_{2}\end{array}\right]=\left[\begin{array}{c}\frac{\Delta}{L} \\ \frac{\Delta\left(C R_{L}+r_{C}\right)}{L C\left(R_{L}+r_{C}\right)}\end{array}\right]$

$C=\left[\begin{array}{ll}0 & 1\end{array}\right]$

In this work, the Buck converter power circuit specifications at a frequency of $80 \mathrm{kHz}$, which are taken from [3, 4], are listed in Table 1.

Table 1,

The parameter values of the Buck converter model $[3,4]$.

\begin{tabular}{llll}
\hline Description & Symbol & Value & Unit \\
\hline Inductance & $\mathrm{L}$ & 47 & $\mu \mathrm{H}$ \\
Capacitor & $\mathrm{C}$ & 68 & $\mu \mathrm{F}$ \\
$\begin{array}{l}\text { Load Resistance } \\
\text { Inductor Effective }\end{array}$ & $\mathrm{R}_{\text {Load }}$ & 2.345 & $\Omega$ \\
$\begin{array}{l}\text { Series Resistance } \\
\text { Capacitor Effective }\end{array}$ & $\mathrm{r}_{\mathrm{L}}$ & 0.13 & $\Omega$ \\
$\begin{array}{l}\text { Series Resistance } \\
\text { The ON-n-Channel }\end{array}$ & $\mathrm{r}_{\mathrm{C}}$ & 55 & $\mathrm{~m} \Omega$ \\
$\begin{array}{l}\text { Resistance of MOS } \\
\text { Transistors }\end{array}$ & $\mathrm{rs}_{\mathrm{on}}$ & 2.1 & $\Omega$ \\
Supply Voltage & $\mathrm{V}_{\text {Supply }}$ & 3.75 & $\mathrm{~V}$ \\
\hline
\end{tabular}




\section{Modified Elman Neural PID Controller Design}

The proposed structure of the closed loop Buck converter PID controller based on a modified Elman neural network technique is shown in Figure (2).

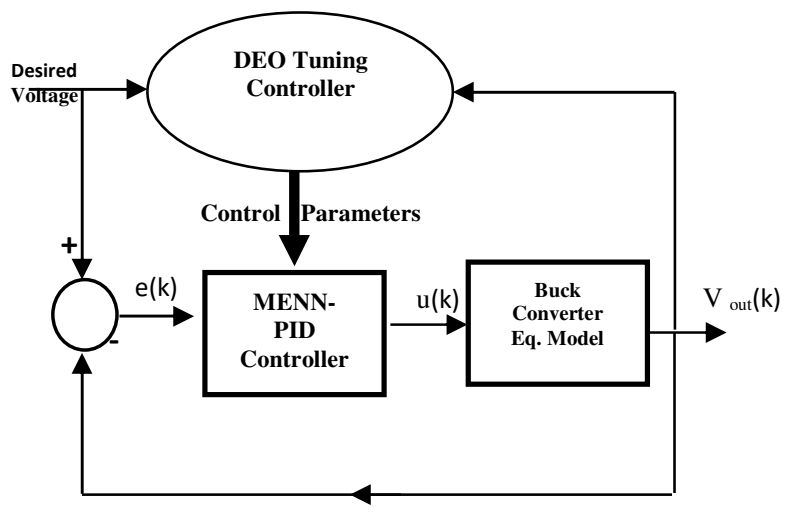

Fig. 2. The structure of MENN-PID controller for Buck converter equation model.

The on-line tune control action of MENN-PID is very essential for stabilizing the error voltage signal of the output of the Buck converter system when the actual output voltage is drifting from the desired voltage and to give a high performance evaluation with disturbance rejection. The PID control equation in the time domain form is given by Eq. (9) $[8,9]$.

$u(t)=k_{p} e(t)+k_{i} \int_{0}^{t} e(t) d(t)+k_{d} \frac{d e(t)}{d t}$

where: $k_{p}$ is the proportional gain; $k_{i}$ is the integral gain; $k_{d}$ is the derivative gain; $u(t)$ is the control action and $e(t)$ is the error signal.

Figure 3 shows the new structure of the MENNPID controller which is constructed from four layers each of which has its own operation as explained below [10]:

- Input Layer: it works as a buffer i.e. pass the data without any modification.

- Hidden layer: it is the active layer with the non-linear activation functions.

- Context layer: it is a memory layer without activation functions.

- Output layer: it represents a linear collector unit which adds all fed signals.

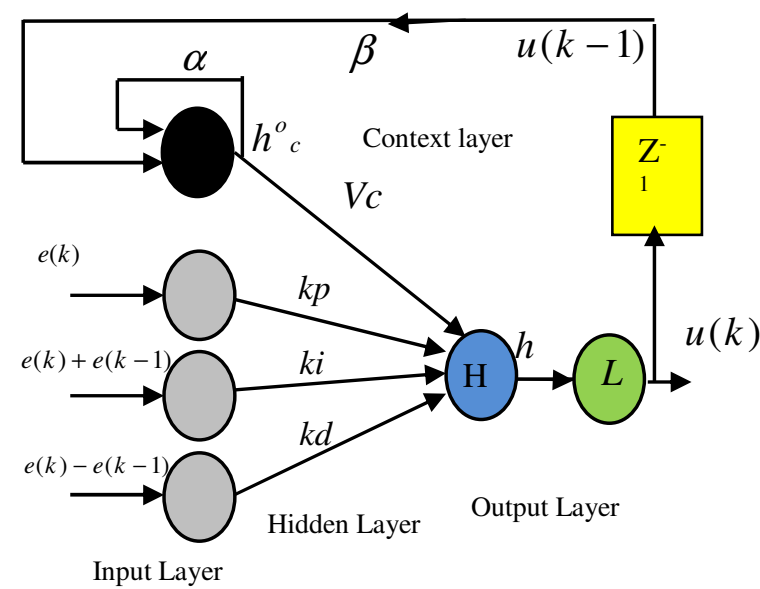

Fig. 3. The new structure of MENN-PID controller.

The network weights notation are: $k p, k i$, and $k d$ hidden layers weights. $V c$ :Context layers weight.

$L i$ : Linear node as scaling factor and it is equal to 1. $H$ : Sigmoid nonlinear node. $h_{c}^{o}(k)$ : Context unit output. $h(k)$ : Hidden unit. $\alpha$ : Selfconnections feedback gain, which is represented randomly between ( 0 to 1$). \quad \beta$ : Connection weight from the hidden layer to the context layer and it is represented randomly between (0 and 1). $e(k)$ : Input error signal. $u(k)$ : Control action signal.

The proposed control law of the MENN-PID controller for the Buck converter system is as follows:

$u(k)=L i \times h(-)$

For the output $h(-)$ of the neural network and it uses a sigmoid activation function as in equation (11) [9]:

$h(-)=\frac{2}{1+e^{- \text {net }(-)}}-1$

net $(-)$ is calculated from equations (12) and (13):

$$
\begin{aligned}
& n e t(-)=(k p \times(e(k)+k i \times(e(k)-e(k-1) \\
& +k d \times(e(k)-e(k-1))+\left(V c \times h_{c}^{o}(k)\right)
\end{aligned}
$$

Where:

$h_{c}^{o}(k)=\alpha h_{c}^{o}(k-1)+\beta u(k-1)$

The new proposed MENN-PID has considerable properties which can be summarized by :

- Fast learning, high adaptation performance, and high order control performance due to the context units in MENN which memorize the previous activations of the hidden units. 
- Good dynamic characteristic, no output oscillation and strong robustness performance due to the self-connection in the context units which increase the order of the controller model.

The near optimal weights of the MENN-PID controller $k p, k i, k d$, and $V c$ will be updated online by using Dolphin Echolocation Optimization algorithm as explained in the next section.

\section{Dolphin Echolocation Optimization Algorithm}

Dolphin echo optimization is a recent discrete meta-heuristic optimization algorithm developed by Ali Kaveh [7]. They inspired it from the fact that the dolphins take the advantages of echolocation to discover the environment and hunt prey in nature the probability of dolphin hunting is increased every time till it gets the prey. The DEO simulates the process of hunting thus, the algorithm proportionally limits (decreases) the exploration space with respect to the target's distance by controlling the index of convergence factor $\mathrm{CF}$ which is defined as the average probability of the best answer. Simply the DEO algorithm divides it work into two phases: in the first phase the algorithm performs a global search and this means that the DEO explores all around the search space by exploring some random locations and in the second phase, the DEO concentrates the search around better results achieved in the first phase i.e. the algorithm goes gradually to the local search in a user defined curve. It is very important to mention here that the probability of getting the near optimal solution is increased in each step ahead till reaching the target $[7,11]$.

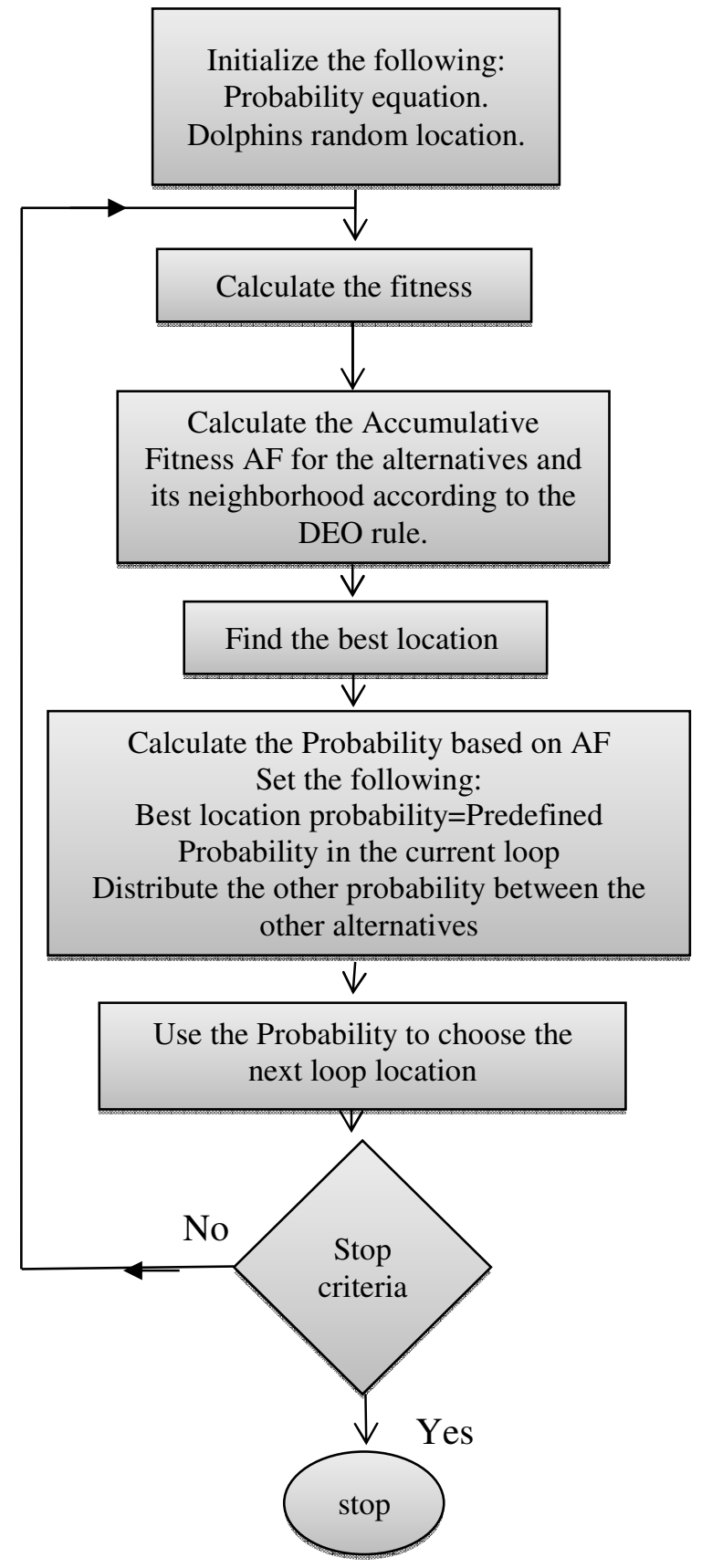

Fig. 4. The flowchart of DEO.

The typical flowchart of the DEO algorithm is shown in figure (4) and the steps of the tuning algorithm are as follows:

\section{Step One "Initialization"}

This step contains the initialization for the following:

- Random Location Matrix L with dimension $\mathrm{L}[-]_{\mathrm{NLXNV}}$ where: NL :number of location; NV number of variable which are $\mathrm{kp}, \mathrm{ki}, \mathrm{kd}$ and $\mathrm{Vd}$ in the proposed controller design. 
- Alternative matrix with dimension [MAxNV] where MA is maximum Alternative number in the search space with ascending order form.

- Maximum loops number $\mathrm{N}$ :number of loops $\mathrm{i}^{\text {th }}$ in which the algorithm should reach to the target.

\section{Step Two "CF predefining and finding"}

Set $\mathrm{PP}_{1}=0.1$ which represent the convergence factor of the randomly selected location in the first loop then use it to find the predefined probability PP according to the equation (14) [7]: $\mathrm{PP}\left(\mathrm{Loop}_{\mathrm{i}}\right)=\mathrm{PP}_{1}+0.1\left(\left(\right.\right.$ Loop $\left._{\mathrm{i}}-1\right)$

Step Three "Fitness calculation"

$\operatorname{Fitness}(\mathrm{Li})=\frac{1}{\operatorname{MSE}(\mathrm{Li})+\mu}$

where $\mu>0$,

MSE: Mean Square Error of location is

$\operatorname{MSE}(\mathrm{Li})=\frac{1}{N} \sum_{i=1}^{N}(\text { Vdes }- \text { Vout })^{2}$

\section{Step Four "Accumulated Fitness AF computation"}

In this step, the search space (alternative matrix) has to be divided into two regions, including an affected region within the affected radius Re and the not affected region. The recommendation says that $\mathrm{Re}$ is a quadric search space.

Now:

- Find the position of the location $\mathrm{L}(\mathrm{i}, \mathrm{j})$ in the $\mathrm{j}^{\text {th }}$ column of the alternative and put it in a vector named A.

note: In $\mathrm{Re}$, the accumulative fitness of the alternative A's neighbors is affected from its fitness then calculate the AF for each $\mathrm{j}^{\text {th }}$ variable in $\mathrm{L}(\mathrm{i}, \mathrm{j})$ location by using the dolphin equation given below [7]:

$$
\begin{aligned}
& A F(A+x)_{i+1}=A F(A+x)_{i}+ \\
& \left\{\begin{array}{cc}
\frac{F i t n e s s(L i)}{R e} & \text { Lower Value }<x \leq L(i, j) \\
\frac{\text { Fitness }(L i)}{R e} & L(i, j)<x \leq \text { Upper Value } \\
0 & x>\text { Upper Value }
\end{array}\right.
\end{aligned}
$$

Where:

Upper Value $=L(i, j)+R e$

Lower Value $=L(i, j)-$ Re

$A+x<0$ and $A+x>L(A j)$ (length of $A j$ ) are not valid.

\section{Step Five "Best location finding"}

Best location will have best AF thus, here terminate algorithm if termination criteria $\mathrm{e} \leq$ $\mathrm{e}_{\text {small }}$ is satisfied, else:

- Find the alternative assigned to the variable of best location

- Let $\mathrm{AF}$ for best location alternative $=0$
Step Six" probability determination and allocation"

- Determine the probability as equation (20).

$P(i, j)=\frac{A F i j}{\sum_{i=1}^{L A j} A F i j}$

- Assign probability equals to PP for all variables of the best location $(\mathrm{P}(\mathrm{i}, \mathrm{j})=\mathrm{PP})$ and $\mathrm{P}(\mathrm{i}, \mathrm{j})=\left(1-\mathrm{PP} \mathrm{P}_{\text {loopi }}\right) \mathrm{P}(\mathrm{i}, \mathrm{j})$

\section{Step Seven "Next loop location selection"}

Update location value with respect to the allocated probability of its alternative.

\section{Step Eight "Repetition"}

Repeat the steps two to seven till the maximum number of iterations is satisfied.

In this work, the steps of the on-line DEO algorithm for finding and tuning control parameters of the proposed MENN-PID controller are repeated at $3.6 \mu$ second (sampling time) for each sample based on Shannon theorem.

\section{Matlab Simulation Results}

The on-line Dolphin Echolocation optimization tuning control algorithm for the proposed MENN-PID controller is carried out by using the MATLAB simulation package in order to achieve the reference output voltage for the DC Buck converter system. By applying the Shanon theorem, the sampling time of the Buck converter system is equal to $3.6 \mu \mathrm{sec}$ based on the time constant of the system which is equal to $\tau=36.5 \mu \mathrm{sec}$ depending on the natural frequency $w n=2.44 \times 10^{+4} \mathrm{rad} / \mathrm{sec}$ and the damping ratio $\zeta=$ 1.12 of the Buck converter system that have been calculated from Eq. (6).

Figure 5 shows the unit step change open loop response for the output voltage of Buck converter system which has stable response.

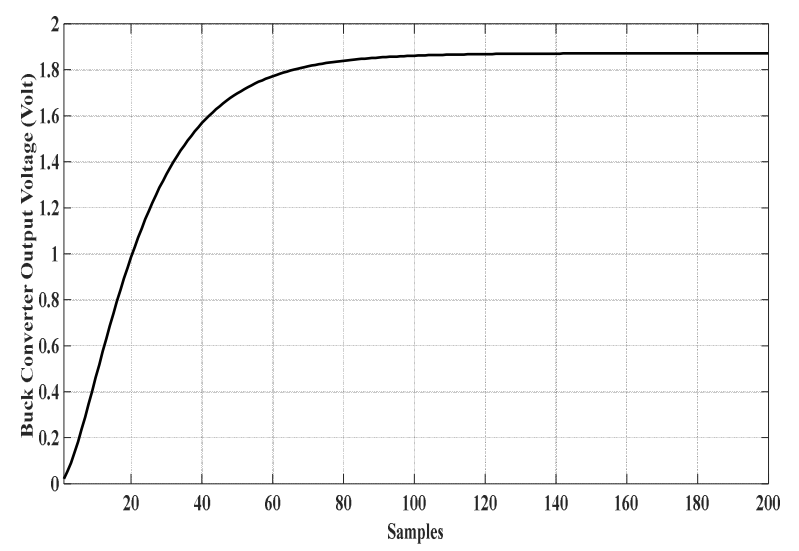

Fig. 5. Open loop response for output voltage of Buck converter system. 
In this work, to investigate the MENN-PID controller as shown in Figure 2 with DEO tuning control methodology which has a capability of generating optimal voltage control action and minimizing the voltage error with minimum number of fitness evaluation, the parameters of the control methodology based on Dolphin algorithm are defined in Table 2.

The number of locations, variables and values of $\mathrm{kp}, \mathrm{ki}, \mathrm{kd}, \mathrm{Vc}$ are selected by using try and error method based on best results that have been obtained.

Table 2,

The parameters of DEO algorithm.

$\begin{array}{ll}\text { The number of locations } & \mathrm{NL}=25 \\ \text { The number of variables } & \mathrm{NV}=4 \\ \text { The best range values of } & \mathrm{kp}=[0.1,0.2,0.3,0.4, \\ \text { kp weight } & 0.5,0.6 \text { to } 4] \\ \text { The best range values of } & \mathrm{ki}=[0.025,0.05,0.075, \\ \text { ki weight } & 0.1 \text { to } 1] \\ \text { The best range values of } & \mathrm{kd}=[0.0125,0.025, \\ \text { kd weight } & 0.0375 \text { to } 0.5] \\ \text { The best range values of } & \mathrm{Vc}=[0.075,0.15,0.225, \\ \text { Vc weight } & 0.3 \text { to } 3] \\ \text { The number of } & \mathrm{MA}=40 \\ \text { alternative matrix length } & \mathrm{N}=10 \\ \text { The number of loop } & \mathrm{Re}= \pm 10 \\ \text { The affected radius } & \mathrm{e} \\ \text { The minimum error value } & \end{array}$

The simulation results of the closed loop voltage control system with the variable step change as (2, 2.5 and 3$)$ volt in the Buck converter's reference output voltage with on-line tuning MENN-PID controller with initial output voltage of the system equals to zero volt can be shown in Figures $(6-a, b, c)$.

The output voltage response of the Buck converter system in the variable step change was very small over-shoot in the transient state while at steady-state, the error was equal to zero value in each step change, as shown in Figure (6-a) when we compared with another controller results as in [4].

In Figure (6-b), the voltage error signal of the closed loop Buck converter controller system was a small value in the transient and it has become zero at the steady state. The action response of the MENN-PID controller was smooth without oscillation response, no spikes behavior and the action did not reach to the saturation state of 3.75 volt depending on the supply voltage. Figure (7) clearly shows the improved performance index of the Buck system based on the Mean Square Error
(MSE) for the on-line tuning Dolphin control methodology at 300 samples.

Figures $(8-a, b, c, d)$ show the parameters of the MENN-PID controller kp, ki, kd and Vc which have been tuned on-line based on Dolphin algorithm at each sample.

In this on-line DEO algorithm, at sample 80 for example, the Accumulative fitness of the $\mathrm{kp}$, ki, $\mathrm{kd}, \mathrm{Vc}$ control gains in the $10^{\text {th }}$ loop or (final loop) of the algorithm can be shown in Figure (9a,b,c,d) respectively.

(a)

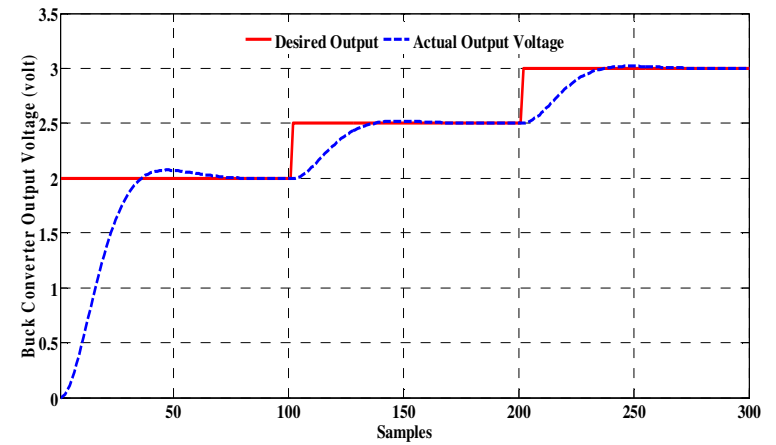

(b)

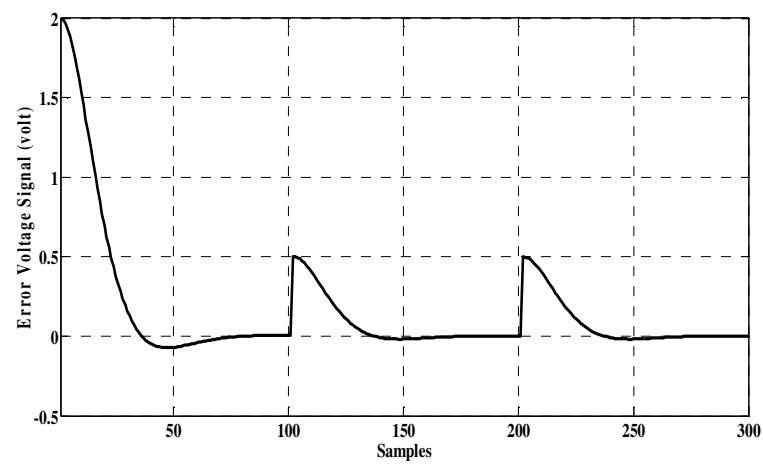

(c)

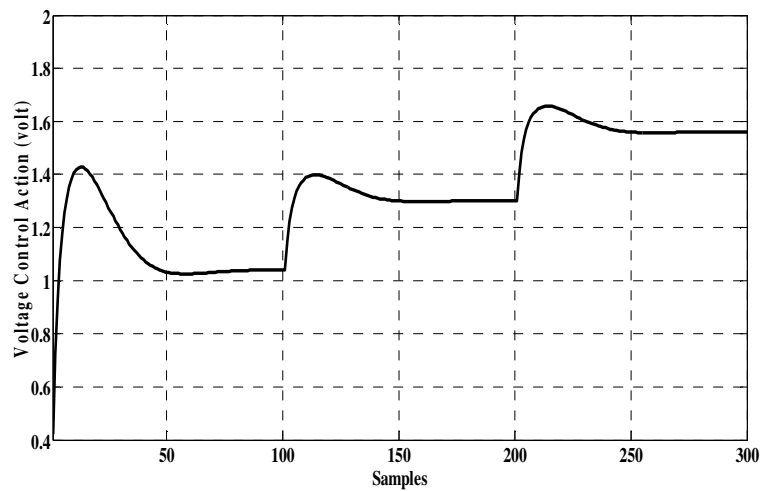

Fig. 6. Simulation results of the adaptive PID controller: (a) Output voltage for Buck converter model; (b) Voltage error; (c) Voltage control action. 


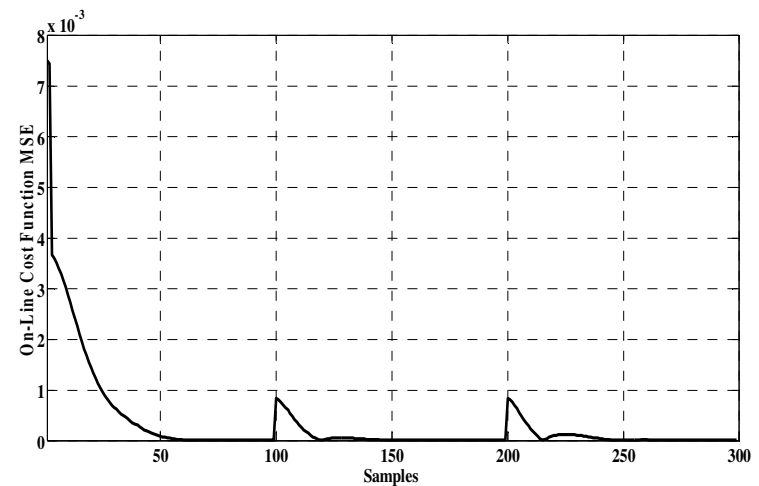

Fig. 7. On-line performance index (MSE).

To verify that the closed loop control Buck converter system has a robust performance for boundary disturbance through adding variable load resistance by decreasing $10 \%$ from its value at sample $100(0.36 \mathrm{msec})$, the output voltage response of the Buck converter system to a step change 1.75 volt has followed the reference voltage at sample $50(0.18 \mathrm{msec})$, the output voltage response of the Buck converter system to a step change 1.75 volt is followed the reference voltage at sample $50(0.18 \mathrm{msec})$ with no overshoot at transit response and robustness response during adding the disturbance as shown in Figure (10-a), the output voltage of the Buck converter system did not accessed \pm 0.05 volt from the reference output voltage and very small overshoot in the transit response then at steady-state response the error voltage signal was equal to zero value.

(a)

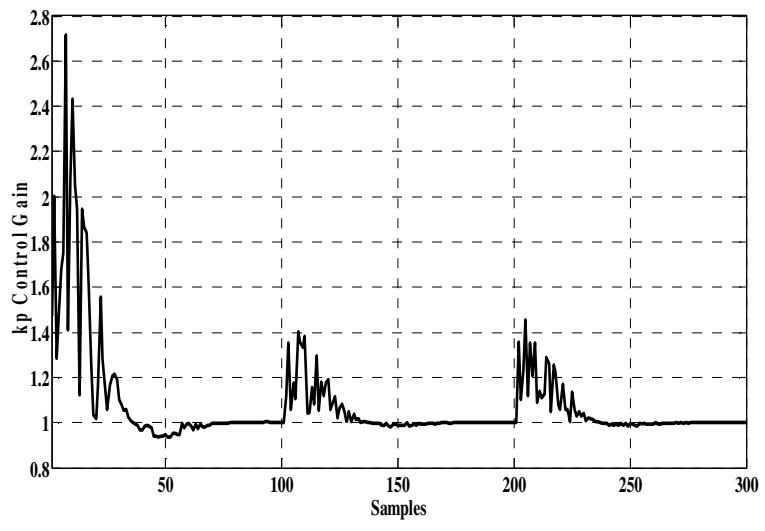

(b)

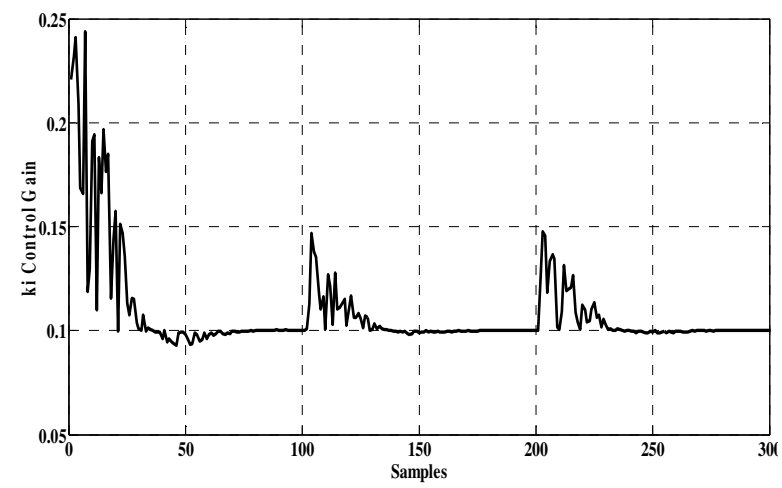

(c)

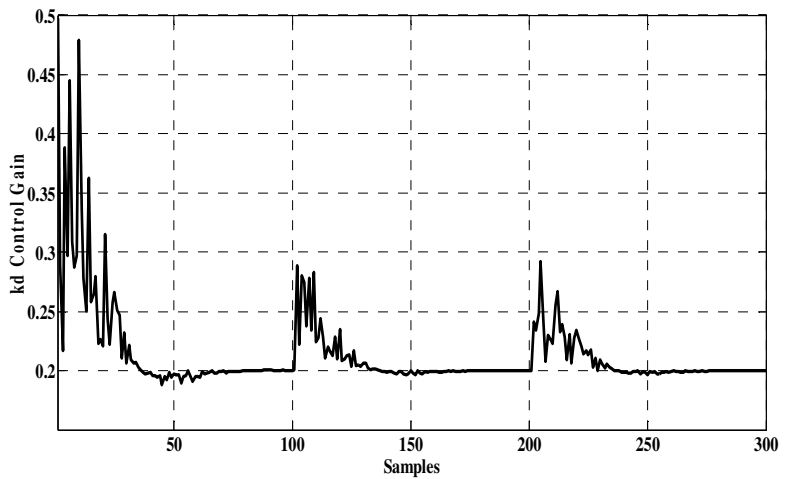

(d)

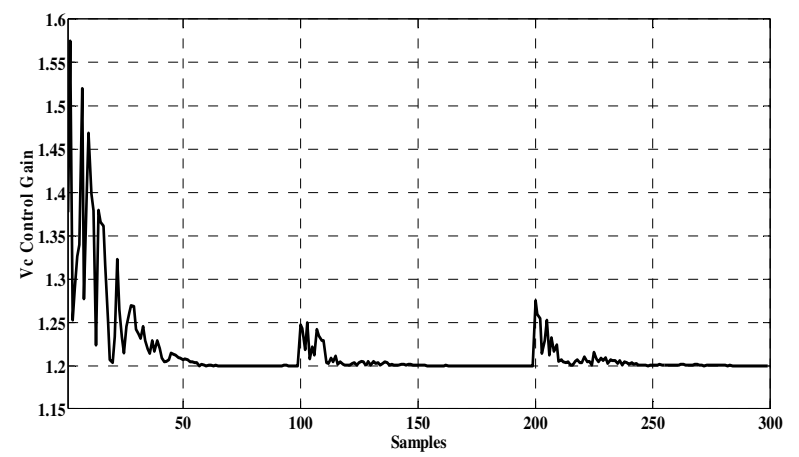

Fig. 8. Simulation results of the control parameters: (a) kp; (b) ki; (c) kd and (d) Vc.

The small value of the error voltage signal between the reference voltage and the output voltage of the Buck converter system is shown in Figure (10-b). Figure (10-c) shows the response of the control action which has a fast and strong adaptability with robustness performance because the control parameters are learned and tuned by a powerful on-line Dolphin algorithm especially when adding boundary disturbance to show the control action has a capability to track the error voltage signal of the Buck converter system to follow the reference voltage as step change and reduce the effect of the load resistance disturbance. 
(a)

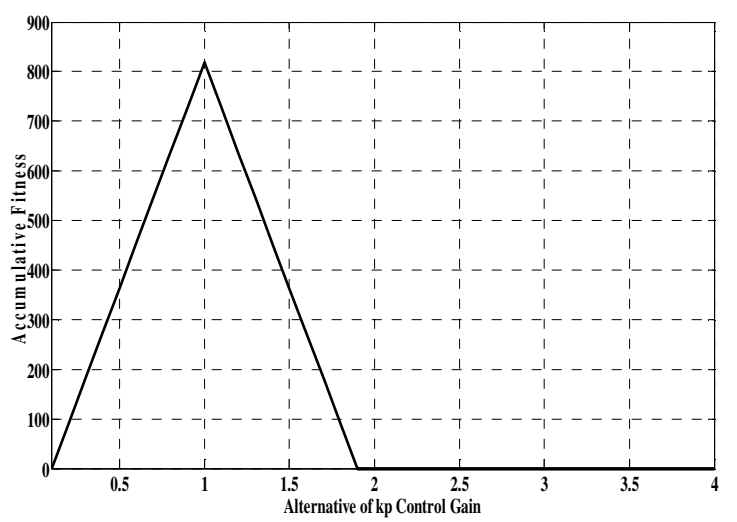

(b)

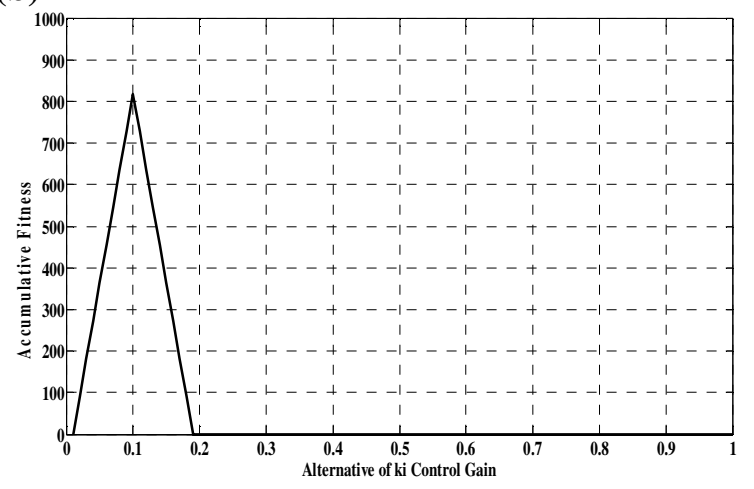

(c)

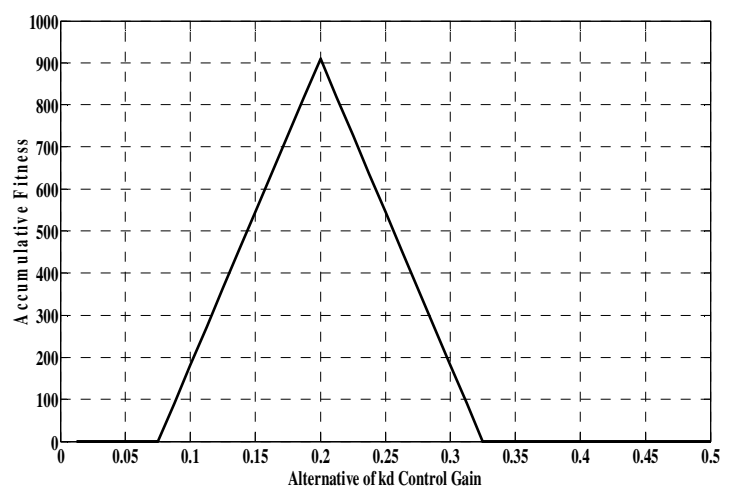

(d)

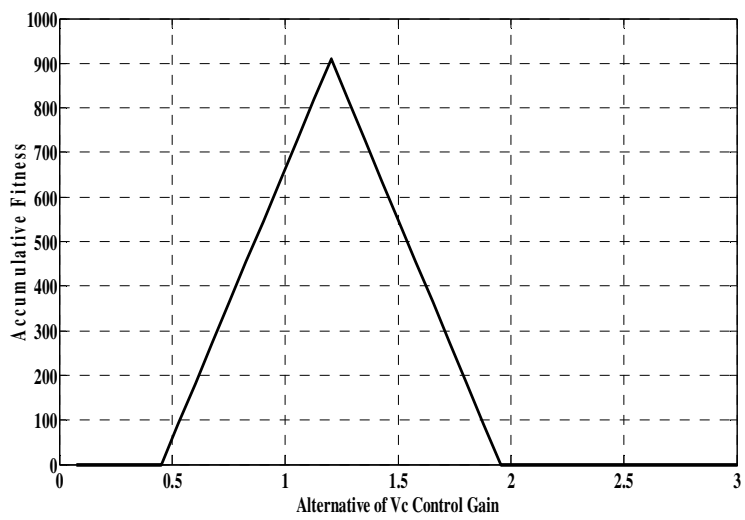

Fig. 9. The Accumulative fitness of the control gains: a) kp, b) ki, c) kd, d) Vc.

Figure 11 shows the cost function of the online tuning Dolphin control algorithm is clearly improved the performance of the proposed MENN-PID controller for the Buck converter system especially at sample 100 during adding disturbance.

The on-line tuning the parameters of the MENN-PID controller $\mathrm{kp}, \mathrm{ki}, \mathrm{kd}$ and $\mathrm{Vc}$ are shown in Figures (12-a, b,c, d) respectively which are found and tuned by using DEO algorithm in order to find the best voltage control action that depends on the initial parameters of the DEO algorithm. 
(a)

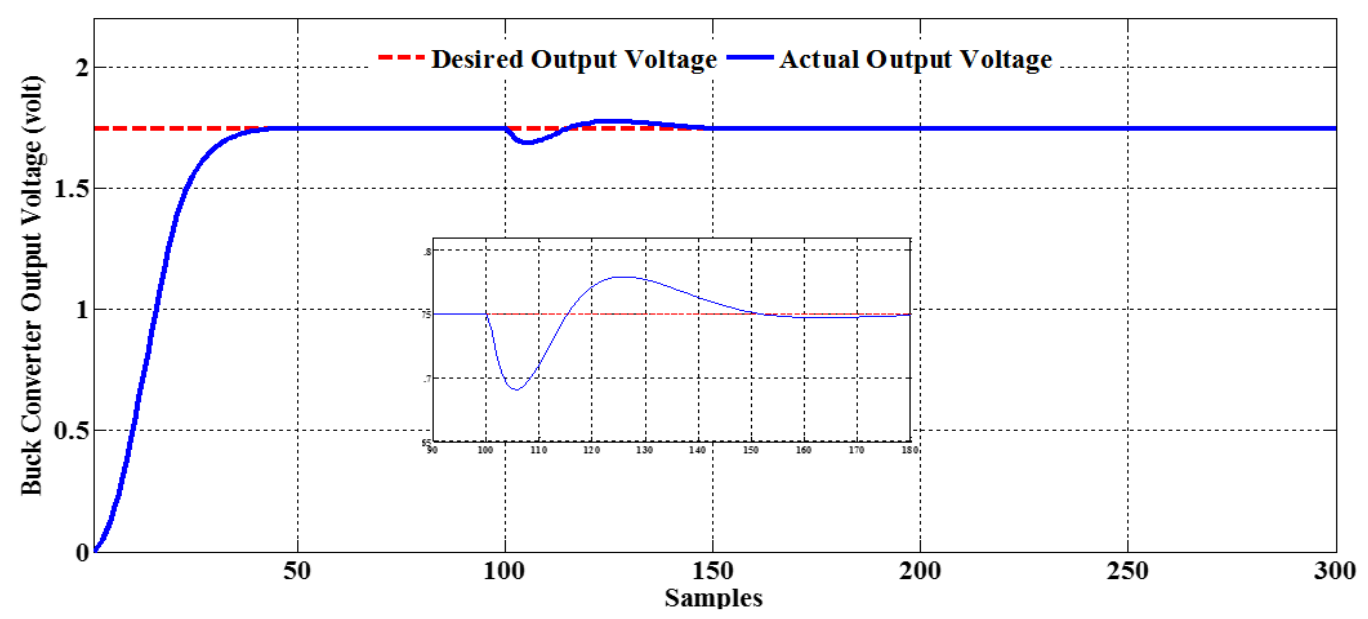

(b)

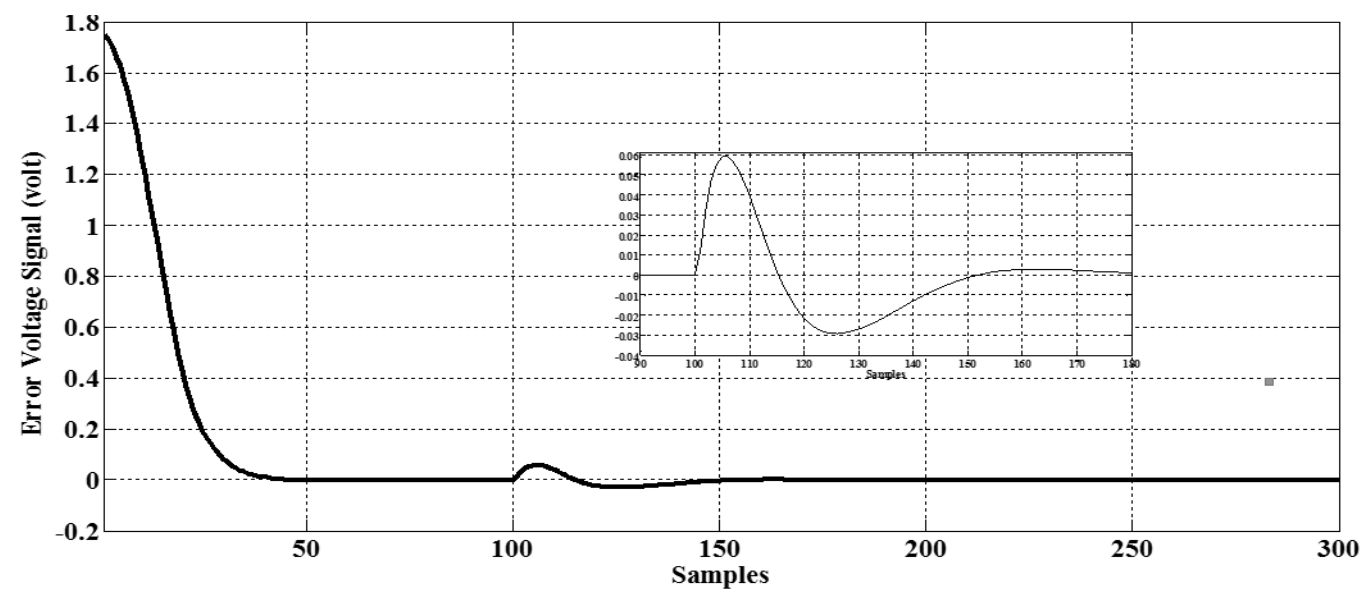

(c)

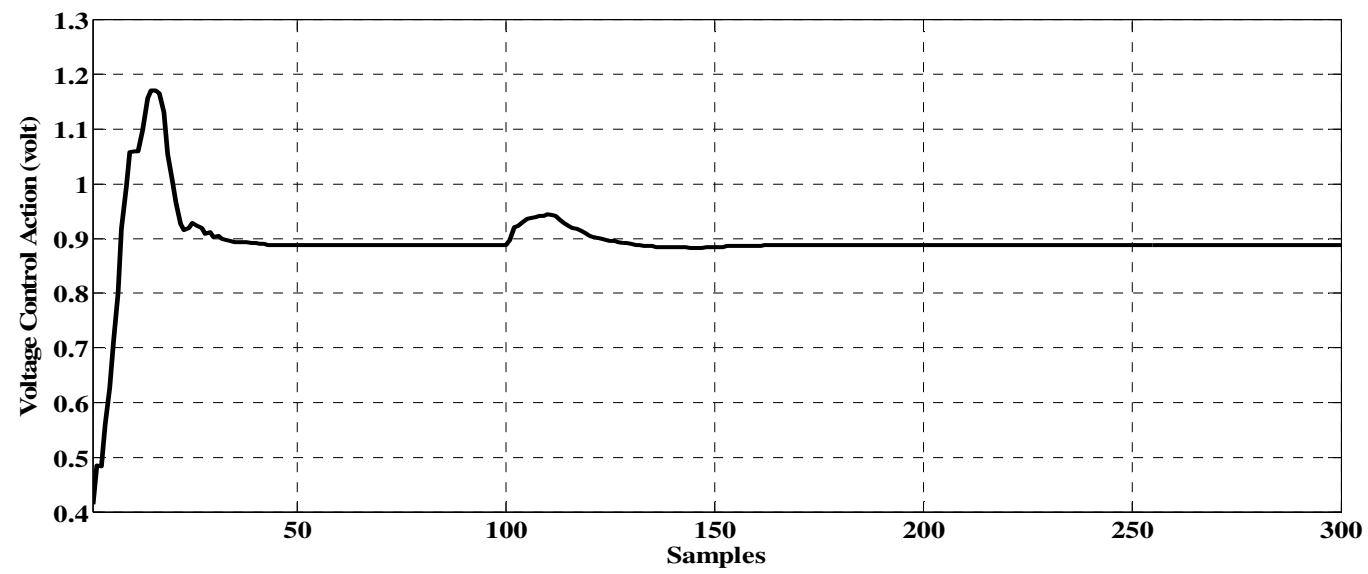

Fig. 10. Simulation results of the MENN-PID controller with disturbance effect: (a) Output voltage for Buck converter model; (b) Voltage error; (c) Voltage control action. 


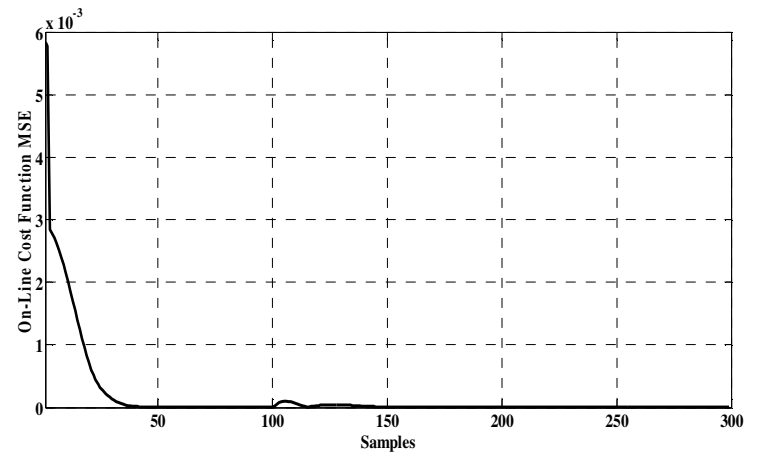

Fig. 11. On-line performance index (MSE) with disturbance effect.

(a)

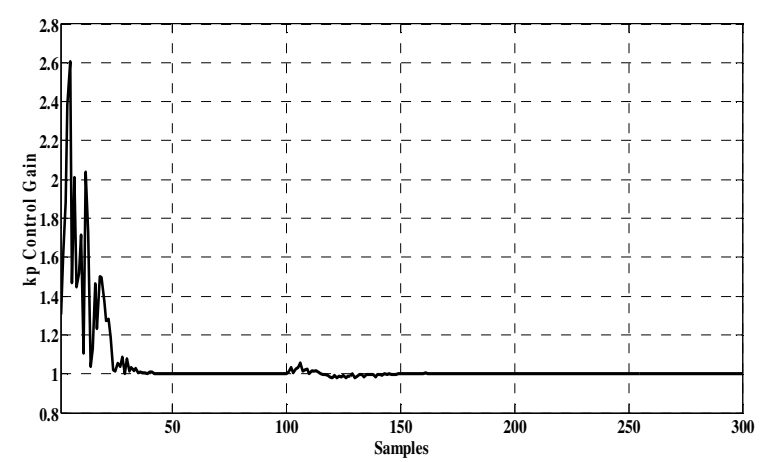

(b)

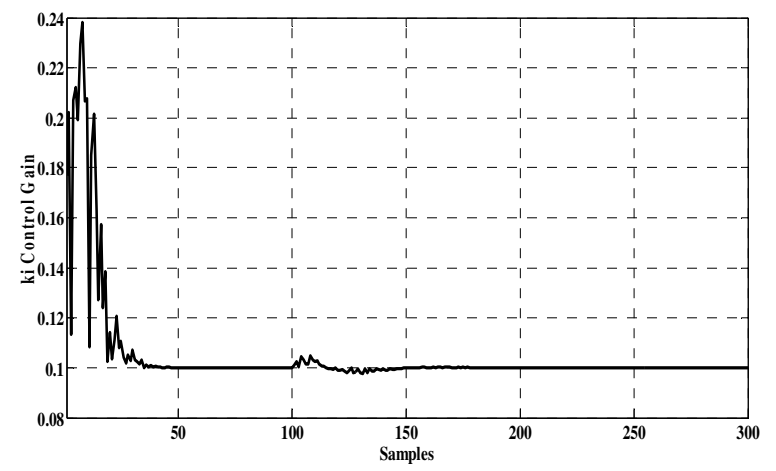

(c)

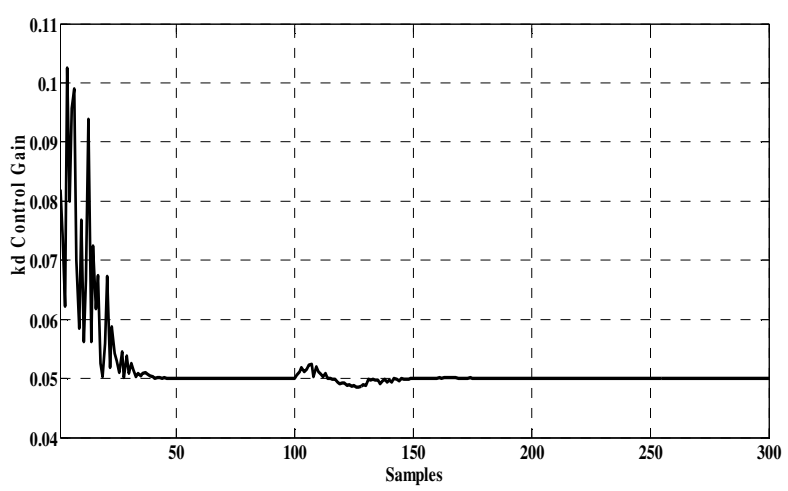

(d)

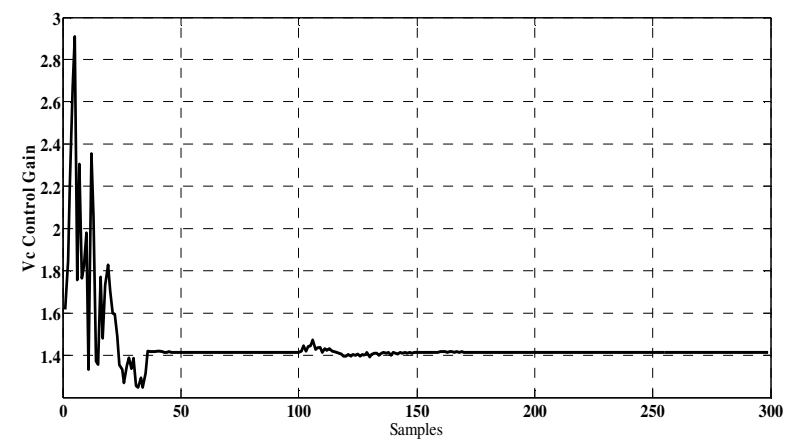

Fig. 12. Simulation results of the control parameters: (a) kp; (b) ki; (c) kd; (d) Vc.

\section{Conclusions}

The numerical simulation results of the proposed MENN-PID controller based on the DEO algorithm is presented in this paper for the DC-DC Buck converter system showing the following capabilities:

- An on-line optimization was achieved for the control gains of the proposed controller.

- A suitable voltage control action was obtained without a saturation voltage state.

- Tracking an excellent reference voltage with a minimum voltage error was achieved.

- Strong adaptability performance through changing the desired voltage level output.

- High robustness performance when changing the load resistance as the disturbances effect to the Back converter system.

\section{References}

[1] S. Chander, P. Agarwal, I. Gupta, "ASIC and FPGA based DPWM Architectures for Single-Phase and Single-Output DC-DC Converter: A Review". Central European Journal of Engineering. Vol. 19, No. 4, pp. 620-643, (2013).

[2] A. Emami, M. Poudeh, S. Eshtehardiha, "Particle Swarm Optimization for Improved Performance of PID Controller on Buck Converter". The International Conference of IEEE on Mechatronics and Automation. pp. 520-524, (2008).

[3] M. Garg, Y. Hote, M. Pathak, "Design and Performance Analysis of a PWM DC-DC Buck Converter Using PI-lead Compensator". 
Arabian Journal for Science and Engineering . Vol. 40, No. 12, pp. 3607-3626, (2015).

[4] M. Tapou, H. Al-Raweshidy, M. Abbod, M. Al-Kindi, "A Buck Converter for DVS Compatible Processors in Mobile Computing Applications Using Fuzzy Logic Implemented in a RISC Based Microcontroller". The $2^{\text {nd }}$ Internation Conference on Circuit and System Control Signal. Prague, Czech. pp. 135-139, (2011).

[5] K-H Kim, B-S Kong, Y-H Jun, "Adaptive Frequency-Controlled Ultra-Fast Hysteretic Buck Converter for Portable Devices". International Conference of IEEE on SoC Design. pp. 5-8, (2012).

[6] J. Chuang, H. Chou, "An Efficient Fast Response Hysteresis Buck Converter with Adaptive Synthetic Ripple Modulator". The $8^{\text {th }}$ International Conference of IEEE on Power Electronics. pp. 620-627, (2011)

[7] A. Kaveh and N. Farhoudi,"A New Optimization Method: Dolphin Echolocation". Advances in Engineering Software. Vol. 59, pp. 53-70, (2013).

[8] A. S. Al-Araji, "Development of an On-Line Self-Tuning FPGA-PID-PWM Control Algorithm Design for DC-DC Buck Converter in Mobile Applications". Engineering Journal. Vol. 23, No. 8, pp. 84106, (2017).
[9] K. E. Dagher and A. S. Al-Araji, "Design of a Nonlinear PID Neural Trajectory Tracking Controller for Mobile Robot based on Optimization Algorithm". Engineering \& Technology Journal Vol. 32, No. 4, pp. 973986, (2014).

[10] A. S. Al-Araji, M. F. Abbod and H. S. AlRaweshidy, "Design of a Neural Predictive Controller for Nonholonomic Mobile Robot Based on Posture Identifier". Proceedings of the IASTED International Conference Intelligent Systems and Control (ISC 2011), Cambridge, United Kingdom, pp.198-207, (2011).

[11] A. Kaveh and N. Farhoudi,"'Dolphin Echolocation Optimization: Continuous Search Space". Advances in Computational Design. Vol. 1, No. 2, pp. 175-194, (2016). 


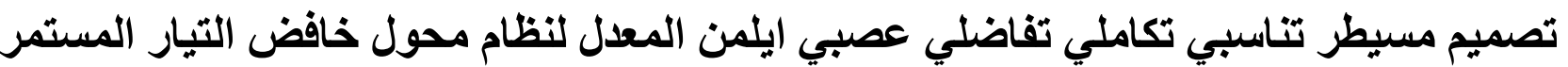

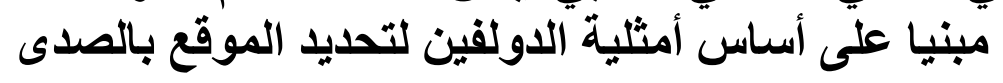

\author{
خلود اسكندر داغر

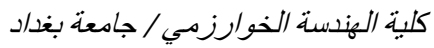

dagherkhulood@kecbu.uobagdad.edu.iq البريد الاككتروني

الخلاصة

إن هذا البحث يصف مقترح جديد لهيكلية المسيطر التناسبي التكاملي التفاضلي بالاعتماد على الثبكة العصبية المعدلة ايلمن لنظام المحول الخافض التضار التيار

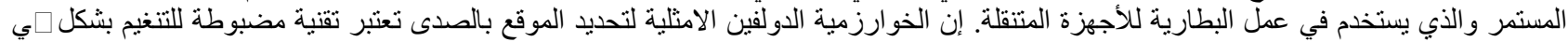

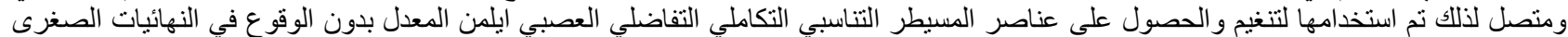

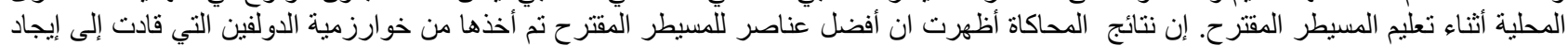

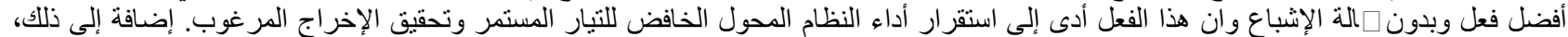

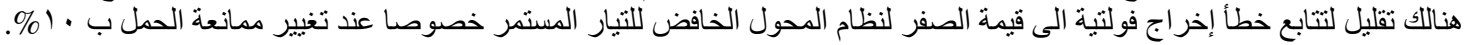

